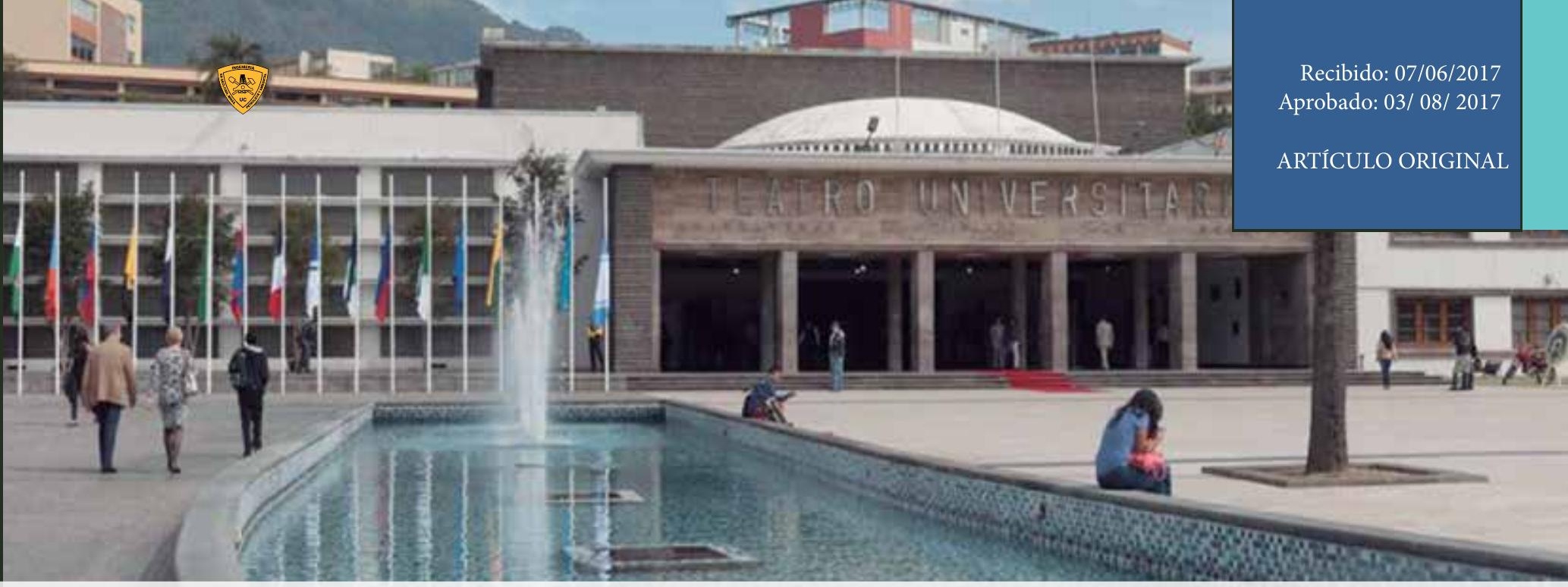

\title{
Portal de datos abiertos de la Universidad Central del Ecuador
}

\section{Open data portal of the Central University of Ecuador}

\section{Cadena Vela, Susana Graciela}

Docente Facultad de Ciencias Administrativas

Universidad Central del Ecuador

scadena@uce.edu.ec

\author{
Enríquez Reyes, Robert \\ Docente Facultad Ingeniería de Ciencias Físicas \\ Universidad Central del Ecuador \\ renriquez@ uce.edu.ec
}

Resumen

La Universidad Central del Ecuador, diariamente genera datos universitarios (estudiantes, docentes y de investigación) que son valiosos para la toma de decisiones y para mejorar la calidad de servicios, sin embargo estos datos no son publicados para que sean utilizados por la comunidad universitaria. Con este propósito el presente documento describe el proceso de desarrollo e implementación del Portal de Datos Abiertos de la Universidad Central del Ecuador (UCE), para fortalecer los procesos de transparencia, participación y colaboración de la comunidad universitaria. El Portal de Datos Abiertos consta de 5 secciones: Inicio, Datos, Aplicaciones, Indicadores y Estadísticas; que se desarrolló de acuerdo a la Metodología Unificada Ágil (AUP), con la finalidad de permitir la sencilla interacción del portal con el usuario. Para ello, se codificó toda su funcionalidad en Liferay e Intellij IDEA. Luego se realizó una conexión a la base de datos de la Universidad con Power BI Desktop, a fin de realizar el proceso de análisis de datos académicos de los estudiantes y docentes para la publicación en el Portal y finalmente la difusión de un conjunto de datos académicos. Con la implementación del Portal se aportó al desarrollo de la transparencia con la publicación en formatos abiertos de los datos generados por la UCE. Estos datos son actualizados y publicados en formatos libres para que el público pueda reutilizar.

Palabras clave: portal web; datos abiertos; gobierno abierto; universidad abierta; liferay; intellij idea; aup

Abstract

The Central University of Ecuador, generates daily university data (about: students, teachers and research), are valuable for making decisions and improving the quality of services. However, these data are no published for use by the university community. That's the reason we develop the Open Data Portal, with the purpose of changing the management of the University to an Open Government, through transparency, participation and cooperation of the university community; which allows the generation of data that can be stored, updated and reused, avoiding the loss of information related to research and innovation projects carried out by both students and teachers. The Central University Open Data Base Portal consists on 5 sections: Menu, Data, Applications, Indicators and Statístics. It was developed according to the Agile Unified Process (AUP), in order to simplify the interaction of the portal with the user. To do this, we codified all its functions in Liferay and IntelliJ IDEA, Then, a connection was made to 
the University database with Power BI Desktop to allow the process of academic data analysis of students and teachers to publish in the Portal, and finally, the publication of academic datasets. With the implementation of the Portal we contributed to the development of transparency with the publication in open formats of the data generated by the UCE. These data are updated and published in free formats o that the public can reuse.

\section{Keywords: web portal; open data; open government; open university; liferay; intellij idea; aup}

\section{Introducción}

Datos Abiertos aparece como un concepto que promete ser muy interesante y que engloba la necesidad de información conjuntamente con su calidad, que de acuerdo con varios investigadores del tema es la forma en que se traducen los datos institucionales a formatos que puedan ser consumidos por diferentes fuentes, es decir, que cualquier persona es libre de utilizar, reutilizar, y redistribuir. [1].

'Datos Abiertos', es un gran recurso, aunque todavía no ha sido explotado para el desarrollo de los gobiernos, el ejemplo más claro es como un gobierno se podría beneficiar cuando los datos están disponibles para diferentes grupos de personas y organizaciones, ocasionando en ellas beneficios y lo más importante participación, no se puede predecir hasta dónde llegará esta nueva tendencia ya que las innovaciones frecuentemente provienen de los lugares más diversos. [1]

Los Datos Abiertos han permitido algunos cambios en los gobiernos que han implementado esta tendencia. Por ejemplo el Gobierno de Canadá ahorró 3.2 millones de dólares que se deducían de manera fraudulenta de los impuestos como donaciones de caridad. Otro ejemplo se encuentra en varios sitios web, como el danés folketsting.dk, donde los ciudadanos pueden seguir las actividades en el Parlamento y el proceso de formulación de leyes para que se pueda ver exactamente qué está pasando y qué Miembros del Parlamento están involucrados. [2]

Los portales de datos abiertos suponen el elemento más visible de las políticas en materia de Gobierno Abierto (Open Government) y una de las principales herramientas de acceso a los recursos informativos del sector público, [3] entonces deberán considerarse como el principal producto tangible en el que se van a ver materializadas las estrategias políticas de las diferentes administraciones públicas en torno al gobierno abierto. Estos portales se han convertido también en una "ventanilla única" que la administración pone al servicio del ciudadano, evitando la dispersión de contenidos entre las diferentes páginas Web de los organismos públicos. [4]

La creación de los portales es muy reciente, encontrándose en una fase de organización y puesta en marcha, por lo que la consistencia y cobertura de los datos es muy desigual. Existe una percepción general de que esta falta de implementación puede deberse a una falta de compromiso real de los gobiernos a impulsar y mantener estas iniciativas, que se contrapone al entusiasmo y dedicación de los responsables directos de cada portal. [4]

Un nivel más alto de implementación conllevará más tareas para "cocinar" los datos antes de ofrecerlos a la sociedad, sin embargo este proceso beneficiará a los ciudadanos porque podrán acceder más fácilmente a los datos para poder reutilizarlos. [5]

Un servicio de datos abiertos no sirve de nada si no se mantiene constantemente actualizado, ampliando continuamente el volumen de información. A más información abierta más potencia tendrán las consecuencias positivas del servicio. [6]

La Universidad Central del Ecuador con una comunidad universitaria de más de 45 mil personas, con una problemática similar en servicios generales de una ciudad, además de los servicios académicos y de investigación que son principal responsabilidad, no dispone de un portal de datos abiertos y adicionalmente, la institución no contaba con la infraestructura tecnológica necesaria para implementar un portal de datos abiertos, no existía conjuntos de datos a ser publicados, ni un procedimiento que garanticen la actualización permanente de esta información, por esta razón se emprende en este proyecto de creación del portal de datos abiertos.

Con estos antecedentes se creó un portal de datos abiertos para que la comunidad de la Universidad Central del Ecuador participe, colabore y visualice información transparente sobre la gestión de la Institución.

En el Portal de Datos Abiertos de la Universidad Central del Ecuador, tiene como finalidad recopilar y actualizar datos académicos y datos de proyectos futuros realizados por estudiantes, los cuales estén disponibles en formato accesible para todo usuario, para que sean reutilizados en futuras investigaciones y mejorar la calidad de servicios que ofrece la universidad, aportando a la generación de conocimiento e innovación [7]. 


\section{Materiales y métodos:}

Para el desarrollo del portal como primer paso se identificaron los riesgos desde etapas iniciales del proyecto utilizando los conceptos que emplea el metodología Unificada Ágil (AUP), [8]. Este proceso establece como premisas fundamentales: "Reducir drásticamente los tiempos de desarrollo pero manteniendo una alta calidad, capturar los cambios para que el cliente tenga una ventaja competitiva, entregar frecuentemente software que funcione en el menor intervalo de tiempo posible entre entregas" [10]. Especialmente el desarrollo de prototipos ejecutables durante la base de elaboración del portal, donde se observe la validez de la arquitectura para los requisitos clave de dicho portal y que determinan los riesgos técnicos.

\section{Consideraciones legales que fueron implementadas}

Para la identificación, recolección, publicación y actualización de Datos Abiertos en el portal web, es necesario tener en cuenta la Ley Orgánica de Transparencia y Acceso a la Información Pública (LOTAIP) y la Constitución Política de la República, ya que la información publicada por la institución no debe vulnerar los derechos de privacidad e intimidad de las personas, que incluye el respeto a las leyes de protección al derecho de autor vigente en el Ecuador. [11]

Para este proyecto se ha tomado en cuenta el modelo de implementación de portal de datos abierto de 5 estrellas desarrollado por Tim Berners Lee, llegando hasta la tercera estrella que permite tener archivos en formato libre [12].

\section{Herramientas usadas para el desarrollo del portal.}

Liferay.- Es un Sistema Gestor de Contenido (CMS por sus siglas en inglés) completo que de acuerdo con la consultora Gartner lidera por varios años cuadrante mágico de CMS, trabaja con lenguaje de programación Java y gestor de base de datos PostgreSQL, los cuales son necesarios según los estándares definidos por la Dirección de Tecnología de la UCE. Además el modelo OSGi que Liferay se adaptó perfectamente a las necesidades de la institución ya que podemos reescribir ciertas características y módulos de Liferay para implementar las funcionalidades que el portal requiere, tal es el caso del módulo de "documents \& media" que fue la parte central para el almacenamiento de los conjuntos de datos (datasets) y la creación de Estructura y Plantillas. [13]

IntelliJ IDEA.- Como entorno integrado de Desarrollo, ya que éste posee una amplia integración con todos los frameworks que se usó en el desarrollo de las Plantillas y Estructuras.

PostgreSQL.- Sistema de gestión de bases de datos de código abierto más potente del mercado, fácil de instalar y configurar. El portal de Datos de Abiertos fue implementado utilizando este gestor ya que la Institución lo definió como estándar para las aplicaciones que alimentan al portal.

Power BI.- Herramienta de Inteligencia de Negocios, que ofrece servicio en la nube y también cuenta con la versión Desktop; que permite al usuario subir, compartir y tener acceso a datos locales, servicios en la nube y orígenes de bases de datos. [14]

Mediante ésta herramienta se conecta directamente a la base de datos de la Universidad Central, para analizar y publicar los datos.

\section{Guía de implementación del portal de datos abiertos}

Para la implementación del Portal de Datos Abiertos de la Universidad Central Del Ecuador se optó por la metodología AUP (Metodología Unificada Ágil), ya que describe un enfoque simple del desarrollo del software usando técnicas y conceptos ágiles. [15]

Fase 1: Iniciación.- En la etapa se identificó el alcance inicial del proyecto, se estableció una arquitectura potencial del sistema, un presupuesto y se buscó el financiamiento, además de la aceptación de las partes interesadas de iniciación de acuerdo con Carvajal, 2013. Esta fase se realizó en base a procesos y necesidades actuales del Portal De Datos Abiertos

Fase 2: Elaboración.- En la fase de elaboración se determinó los factores de riesgo conocidos y se estableció la arquitectura del sistema. Se realizaron algunos procesos comunes tales como: la creación de diagramas de casos de uso, diagramas conceptuales, diagramas de base de datos, como lo recomienda Carvajal, 2013.

Fase 3: Construcción.- En esta fase se construyó el sistema sobre las bases establecidas en la fase de elaboración. Las características del sistema se implementaron en una serie de iteraciones cortas, de tiempos definidos. Los resultados de cada iteración son una versión ejecutable del software. Se elaborarán casos de uso durante la fase de construcción y cada uno se convirtió luego en el inicio de una nueva iteración, según Carvajal, 2013.

Fase 4: Transición.- En esta fase se mostró el sistema a los usuarios finales, se recibieron comentarios y se realizó una retroalimentación de la versión, este proceso dio lugar a ajustes adicionales que se incor- 
poraron a lo largo de varias iteraciones. La fase de transición también incluyó conversiones de sistema y capacitación de los usuarios.

El final de la Fase de Transición se marcó por la entrega de una versión semi funcional del producto que fue paulatinamente construyendo el producto final.

\section{Resultados:}

Inicio-- En la figura 1 se encuentra la presentación del portal, en el cuál el usuario puede acceder a las diferentes opciones del menú y la opción de ingresar o registrarse para poder participar, comentar y calificar en las publicaciones de los datos que ofrece el portal.

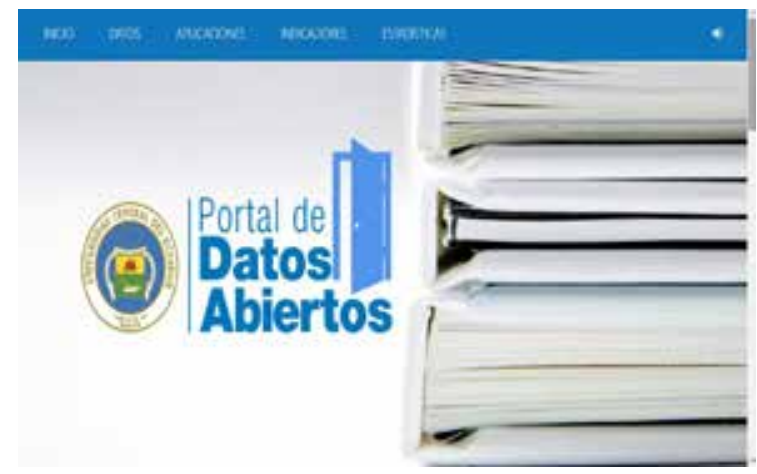

Figura 1: Portal de Inicio.

Datos.- En la figura 2 se presenta el acceso a la búsqueda de datos donde el usuario puede buscar, filtrar, ver los conjuntos de datos más valorados y seleccionar el conjunto de datos que desea ver o descargar.

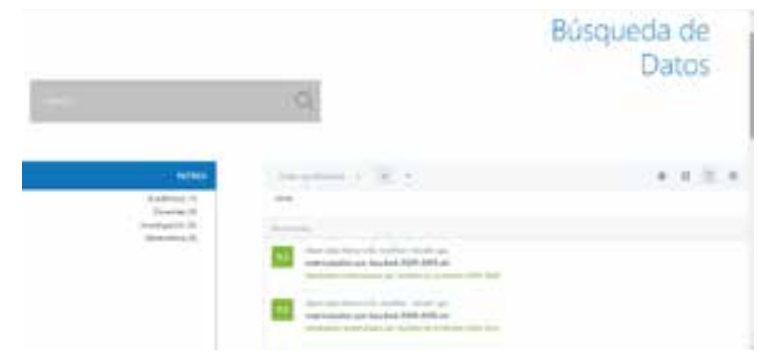

Figura 2: Portal de Búsqueda de Datos.

Aplicaciones.- En la figura aparece la búsqueda de aplicaciones de datos, donde el usuario puede seleccionar la función de buscar, seleccionar la aplicación, calificar, comentar y descargar.

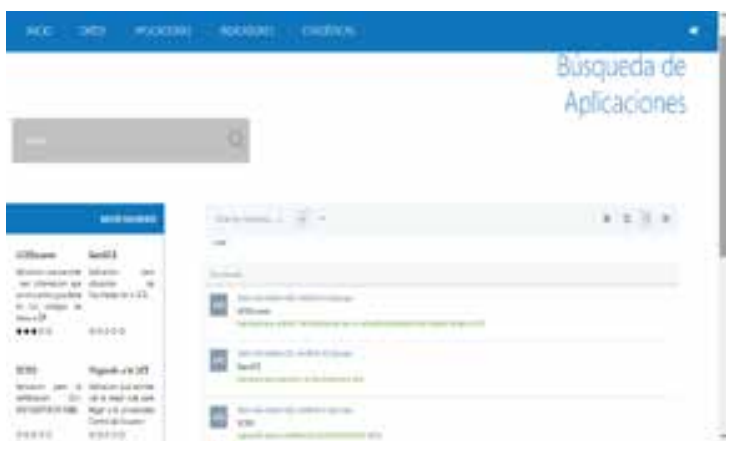

Figura 3: Portal de Aplicaciones.

Noticias.- En la figura 4 presenta el acceso a noticias que permite informar al usuario con noticias relacionadas a datos abiertos, universidad abierta, proyectos relacionados al tema, entre otros.

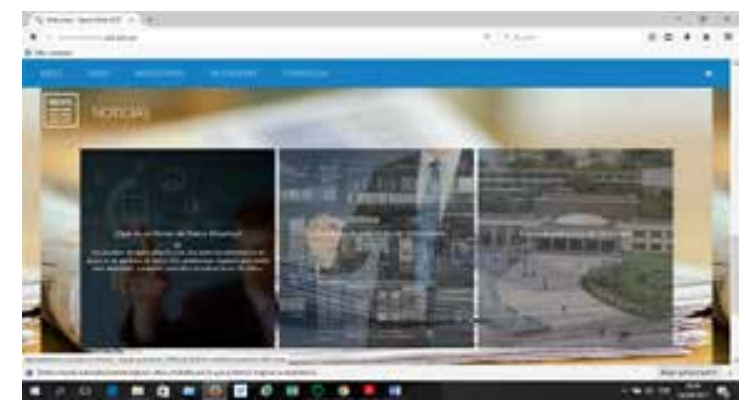

Figura 4: Portal de Noticias.

Contactos. - En la figura 5 se presenta como el usuario puede obtener información del Portal, números telefónicos, enlaces y dirección.

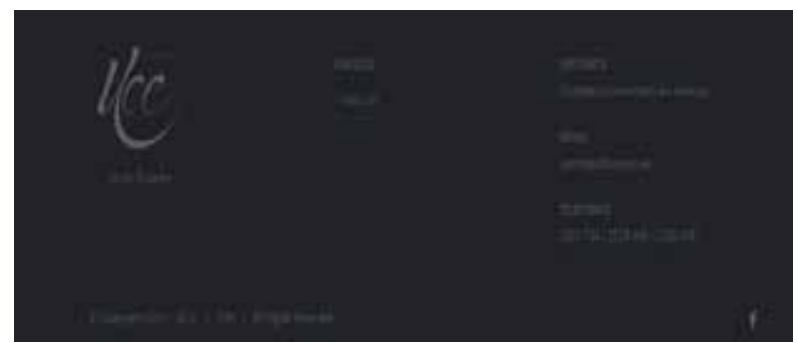

Figura 5: Portal de contactos ESTA FIGURA NO SE VISUALIZA

Estadísticas.- En la figura 6 se presenta cómo los usuarios pueden observar las estadísticas del portal web que son:

- Sesión por día.

- Número de sesiones.

- Sistema operativo del cual se conecta.

- Sesión por país.

- Número de usuarios por fecha.

- Número de usuarios por semana. 


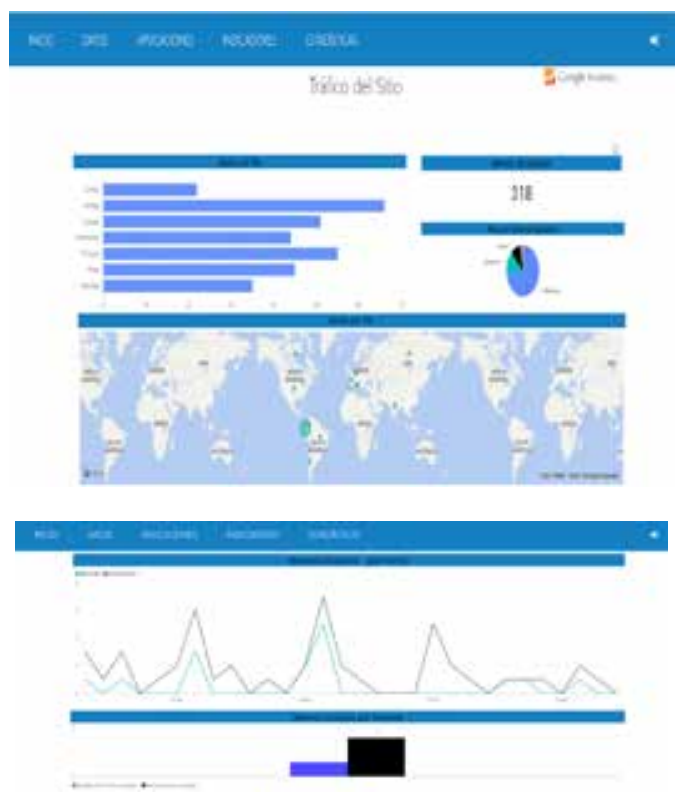

Figura 6: Estadísticas Tráfico del Sitio.

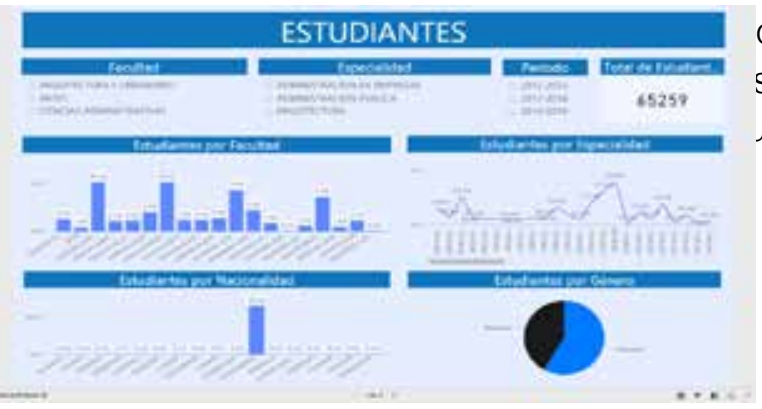

Figura 7: Indicadores Académicos.

Los datos serán actualizados y mantenidos por la Dirección de Tecnología.

\section{Discusión}

Realizado un análisis de las herramientas para la creación de acuerdo a sus beneficios y relaciones de costo / beneficio se puede comprobar que es posible la implementación del portal de datos abiertos con un sistema híbrido entre herramientas libre acceso y herramientas propietarias. Dependiendo del grado de madurez que se desea alcanzar de acuerdo a la metodología de 5 estrellas de Tim Berners-Lee se requerirá mayor desarrollo de software, en el estudio presente se ha alcanzado hasta la estrella 3, donde podemos bajar archivos en formato abierto no propietario para que sean reutilizados.

Una vez implementada la plataforma se empieza a integrar conjuntos de datos de diferentes aplicaciones que permite asegurar la reutilización de los datos, actualmente se tiene los siguientes conjuntos de datos:

- Datos académicos de estudiantes por semestre, en la figura 8 se muestra una parte de los archivos que es posible descargar de la plataforma

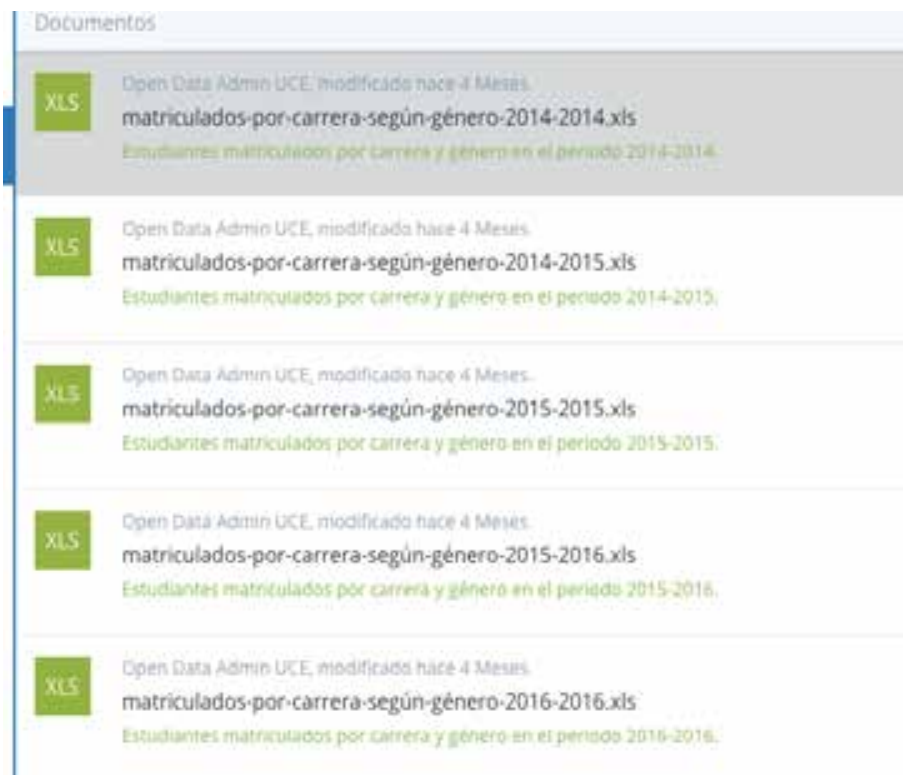

Figura 8. Ejemplos de archivos de datos abiertos de alumnos en diferentes periodos

El proceso de actualización de los datos se realiza en forma manual, generando de la base de datos del sistema académico los archivos de estudiantes matriculados en el periodo requerido.

- Data sets de aplicaciones móviles, generados a través de participación estudiantil en concursos de desarrollo de software, la figura 9 muestra las aplicaciones subidas a la plataforma

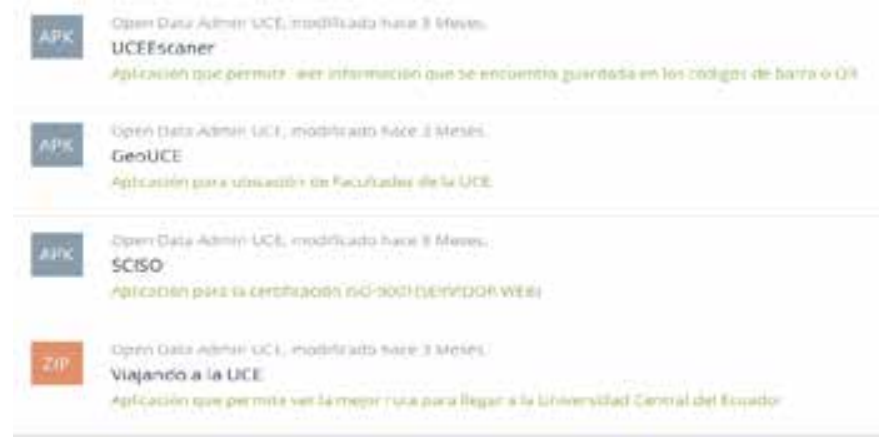

Figura 9. Aplicaciones subidas a la plataforma de datos abiertos.

Las aplicaciones se pueden descargar a los dispositivos móviles a través de google store.

El siguiente reto es lograr una actualización automática de los archivos requeridos, que las aplicaciones se puedan descargar del portal y subir a grado de estrella 4 donde se pueda aplicar la técnica del análisis predictivo de datos por computador y poder reutilizar los datos de manera más rápida con uso de tecnologías de inteligencia artificial.

\section{Conclusiones}

Se creó el Portal de Datos Abiertos de la Universidad Central del Ecuador, con el fin de que la comunidad universitaria pueda reutilizar los datos a través de la generación de proyectos académicos y de investiga- 
Los datos generados pueden ser actualizados y reutilizados, como por ejemplo para la creación de Indicadores académicos.

Se publicó aplicaciones móviles fruto de proyectos realizados por estudiantes de la Facultad de Ingeniería, en formatos que el público puede acceder fácilmente y comprender para ser reutilizados y distribuidos.

Se logró identificar la participación de los usuarios internos y externos a la UCE mediante la opción de "Estadísticas", además el portal permitió registrar a usuarios que querían interactuar, participar y calificar mediante comentarios que permitirán mejorar el portal.

\section{Agradecimiento}

Se agradece a todos quienes participaron en el desarrollo del Portal de Datos abiertos, especialmente al Departamento de Tecnología de la Universidad Central del Ecuador.

\section{Referencias bibliográficas}

[1] W3C, «The World Wide Web Consortium (W3C),» 2908 2012. [En línea]. Available: http://www.w3.org/.

[2] Biblioguías - Biblioteca de Cepal, «Datos Públicos Abiertos,» 1707 2015. [En línea]. Available: http://biblioguias. cepal.org/c.php?g=159545\&p=1044727.

[3] G. Concha y A. Naser, «Datos abiertos: Un nuevo desafio para la region,» CEPAL, 2012.

[4] M. Cuenca y R. Arquero, «El Portal de datos abiertos de la Unión Europea: análisis y evaluación,» Universidad Complutense de Madrid, p. 20, 2014.

[5] F. Ghesquiere, A. Simpson, R. Soden, V. Deparday, J. Crowley y D. York, Open Data for resilience initiative field guide, Washington: The World Bank, 2014.

[6] L. F. Ramos Simón, R. Arquero Aviles, L. Botezan, S. Cobo Serrano, A. Saia Jimmenez, R. Sancez Jimenez y F. d. Valle Gastaminza, «De la reutilización de información del sector público a los portales de datos abiertos en Europa,» textos universitaris de biblioteconomia i documentació, p. 36, 2012.

[7] R. A. Enríquez Reyes y S. G. Cadena Vela, «Diseño e implementación de una Universidad Abierta Caso Universidad Central del Ecuador, "Sexta Conferencia de Directores de Tecnología de Información y Comunicación
[8] T. Vanfosson, «Plan-driven vs. Agile Software Engineering and Documentation: A Comparison from the Perspectives of both Developer and Consumer Submitted for the PhD Qualifying Examination., „ CiteSeerX, p. 20, 2006.

[9] S. Ambler, «The Agile Unified Process (AUP),» 1612 2016. [En línea]. Available: http://www.ambysoft.com/unifiedprocess/agileUP.html.

[10] J. H. Canós, P. Letelier y M. C. Penadés, Métodologías Ágiles en el Desarrollo de Software, Alicante: Grupo ISSI, 2003.

[11] S. N. d. A. P. SNAP, «Datos Abiertos - Sistema Nacional de Información,» 2014. [En línea]. Available: http://sni. gob.ec/documents/10180/410435/GuiaDatosAbiertosv1GPP-DA-v01-20141128-SNAP-SGE.pdf/448eaec9-f738452e-b825-399303e51d70.

[12] T. Berners-Lee, «5 star deployment scheme for Open Data.».

[13] Liferay, «Lifaray como una plataforma de desarrollo,» febrero 2017. [En línea]. Available: https://dev.liferay.com/ develop/tutorials/-/knowledge_base/7-0/liferay-as-a-development-platform.

[14] Microsoft, «Power BI,» 2601 2017. [En línea]. Available: https://powerbi.microsoft.com/es-es/.

[15] P. Carvajal, «Metodologias RUP, UWE, XP, AUP,» 25 octubre 2013. [En línea]. Available: http://trimestrevpstiipedrocarvajaltiv.blogspot.com/.

[16] Liferay, «Liferay,» 12 mayo 2016. [En línea]. Available: https://www.liferay.com/es.

[17] A. Naser y Á. Ramírez Alujas, «Plan de gobierno abierto,» Santiago de Chile, 2014.

[18] W3C, «SEMANTIC WEB,» 2012 2016. [En línea]. Available: https://www.w3.org/standards/semanticweb/.

[19] Liferay, «INTRODUCCIÓN AL DESARROLLO DE LIFE$R A Y, » 10$ febrero 2017. [En línea]. Available: https://dev. liferay.com/develop/tutorials/-/knowledge_base/7-0/introduction-to-liferay-development. 